\title{
Understanding the Conformational Preference of Propeller-shaped Polycyclic Aromatic Hydrocarbons
}

\author{
Alex van der Ham, ${ }^{+[a]}$ Thomas Hansen, ${ }^{+[a, b]}$ Herman S. Overkleeft, ${ }^{[a]}$ Dmitri V. Filippov, ${ }^{[a]}$ \\ Grégory F. Schneider*[a] and Trevor A. Hamlin*[b]
}

[a] A. van der Ham, Dr. T. Hansen, Dr. D. V. Filippov, Prof. Dr. H. S. Overkleeft, and Dr. G. F. Schneider

Leiden Institute of Chemistry, Leiden University, Einsteinweg 55, 2333 CC Leiden (The

Netherlands)

* Email: g.f.schneider@chem.leidenuniv.nl

[b] Dr. T. Hansen and Dr. T. A. Hamlin

Department of Theoretical Chemistry, Amsterdam Institute of Molecular and Life Sciences

(AIMMS), Amsterdam Center for Multiscale Modeling (ACMM), Vrije Universiteit Amsterdam, De Boelelaan 1083, 1081 HV Amsterdam (The Netherlands)

*Email: t.a.hamlin@vu.nl

+ These authors contributed equally to this work

\begin{abstract}
The physico-chemical properties of chiral propeller-shaped PAHs (propellerenes) are strongly dependent on their conformational behavior. A sound, physical model to understand why propellerenes exhibit a conformation preference for either a $C_{2}$ or $D_{3}$ conformation that moves beyond a phenomenological explanation is needed. We have therefore performed a computational study to rationalize the conformational preference of propellerenes. Using an activation strain analysis approach, we find that the conformational preference of propellerenes is ultimately determined by the flexibility of the wings. When wings are relatively flexible, as is the case for ortho-substituted propellerenes, a favorable contraction of the radial bonds connecting the core and the propellerene wings is possible, and the more distorted $C_{2}$ conformation will be preferred. The more rigid wings of benzenoid propellerenes, on the other hand, cannot deform sufficiently, and will therefore always adopt a $D_{3}$ conformation. Our approach represents a unique method to pinpoint the conformational preferences of propellerenes, and, in principle, any sterically congested molecule.
\end{abstract}

Keywords: Polycyclic aromatic hydrocarbons; Conformational behavior; Activation strain model; Density functional theory; Propellerenes 


\section{Introduction}

Non-planar, chiral polycyclic aromatic hydrocarbons (PAHs) exhibit unique photophysical and chirooptical properties. ${ }^{1-6}$ One subclass of particular interest are the helicenes, which are molecules composed of ortho-fused aromatic rings, and that adopt a helical geometry. ${ }^{7-9}$ Although helicenes hold potential for use in chiro-optical applications, low barriers to racemization and poor photo-stability towards UV light, pose significant challenges. Different strategies have therefore been devised to design molecules that incorporate these interesting helicene motives but do not suffer these shortcomings. Apart from increasing the number of fused rings (i.e. $[n]$ helicenes with $n \geq 6$ ), and the introduction of heteroatoms into the helicene structure, another interesting approach to increase the photo-stability is by the combining of two or more helicene units into a double, ${ }^{10-24}$ triple, ${ }^{2,3,25-31}$ or even quadruple ${ }^{32}$ helicene. Unfortunately, the merger of multiple helicene units into a single molecule also changes its physicochemical properties in a non-straightforward manner. ${ }^{33}$ Tuning of these properties has thus far proceeded primarily with a trial-and-error approach. Indeed, despite earlier (computational) endeavors to arrive at a unifying model to rationalize the preferred geometry of these molecules, such a model remains elusive. In this context, the conformational behavior of triple helicenes, which we call propellerenes, has been the topic of much debate. ${ }^{34-39}$

Propellerenes can be regarded as consisting of two integral parts: the six central carbon atoms, which we refer to as the core, and the rings and substituents attached thereto, called the wings (highlighted in green and red, respectively, in Figure 1). What differentiates propellerenes from other helicene derivatives is that the conformational space attainable to them is limited to two distinct conformations, labeled according to their symmetry group as either $C_{2}$ or $D_{3}$. These conformations are dictated entirely by the relative orientation of the wings of the propellerene and are thus the only conformations which are geometrically allowed (Scheme 1). The composition of the wings allows propellerenes to be further divided into two families: wings bearing non-fused substituents are denoted ortho-substituted (Figure 1; 1-4), whereas those with additional fused benzene rings are denoted as benzenoid (Figure 1; 5-8).

a) Ortho-substituted propellerenes

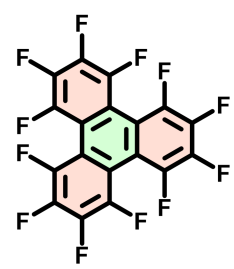

1

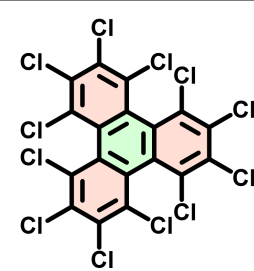

2

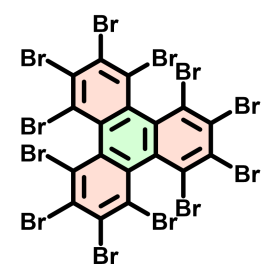

3

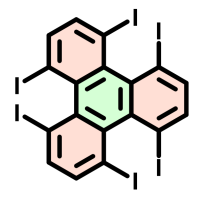

4

b) Benzoid propellerenes

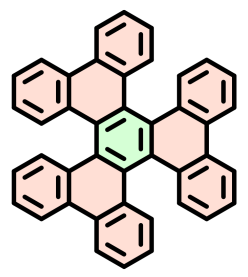

5

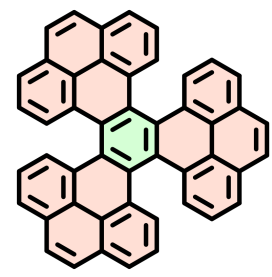

6

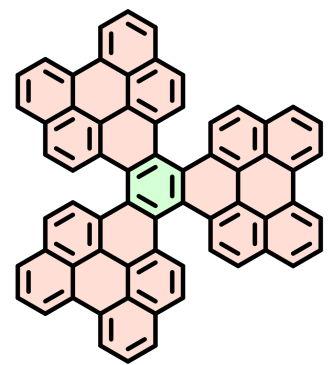

7

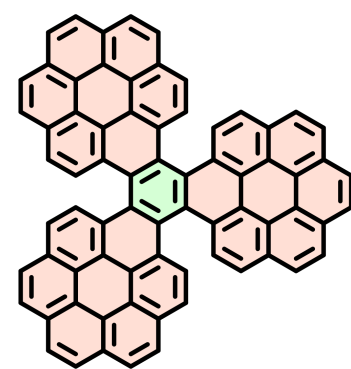

8

Figure 1. Structures of a) ortho-substituted and b) benzenoid propellerenes. Propellerene cores are colored green and the wings colored red. 
Propellerenes typically show a strong preference for either a $C_{2}$ or $D_{3}$ conformation and previous computational and experimental findings have pointed towards a combination of aromatic, steric, and electrostatic factors to be at play in driving this preference. ${ }^{34-39}$ Pascal et al. were the first to systematically study the conformational behavior of propellerenes and proposed that the preferential adaptation of a $C_{2}$ conformation is driven by a greater conjugation of the aromatic systems of the wings, at the expense of that of the core. ${ }^{40,41}$ Although this theory holds for simple, ortho-substituted propellerenes, those with additionally fused benzene rings, i.e. benzenoid propellerenes, defy this general assumption, and show a preference for a $D_{3}$ conformation., ${ }^{2,327,42,43}$ To address this issue, the same group published a largely computational study a few years later, from which they concluded that the $C_{2} / D_{3}$ dichotomy is not driven by steric factors but is a purely electronic effect. ${ }^{35}$
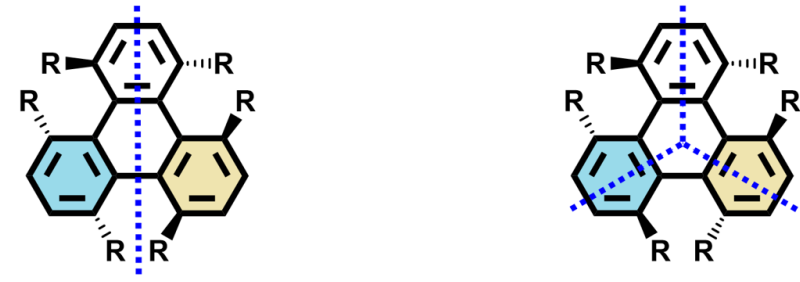

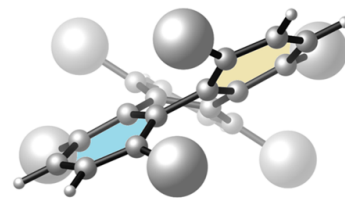

$C_{2}$

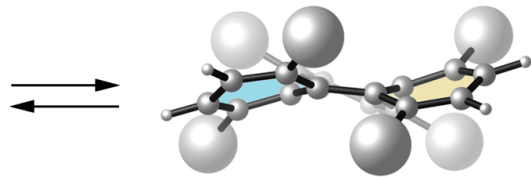

$D_{3}$

Scheme 1. General representation of the $C_{2} \rightleftarrows D_{3}$ interconversion of propellerenes. Note that the rings highlighted in blue and yellow change their orientation relative to the ring at the back. Dashed blue lines represent the axes of rotation which define the symmetry group of the propellerene.

Recent advances in computational chemistry and expansion of the propellerene library prompted us to revisit this topic. Based on newly gained insights regarding the conformational behavior of propellerenes, we hypothesized that the origin as to why some propellerenes prefer to have wings in a $C_{2}$ symmetrical arrangement and others a $D_{3}$ symmetrical arrangement, could be rationalized from the balance in the individual preference of the wings and the core of the propellerene molecule.

To this end, we undertook a computational study to elucidate and comprehensively describe the thermodynamic and kinetic factors that drive the conformational preference of propellerene molecules. We focused on the triphenylene class of propellerenes, which constitutes the most widely studied class of propellerenes. In the ortho-substituted class, halogenated triphenylenes were chosen as the halogen substituents constituted an intrinsic trend in size, with molecules 1, 2 and $\mathbf{4}$ being additionally known experimentally in the literature. For the benzenoid class, molecules 5-8 were selected in which, starting from 4, additional benzene rings are incorporated into the wings, increasing their rigidity. We first started by studying the conformational preferences of propellerenes and their ability to interconvert between their two conformers. Next, to understand the origins of the thermodynamic preference of propellerenes, we employed and adapted the activation strain model (ASM). The present methodology is applicable not only to helicenes, but to non-planar aromatics in general, and will aid in their rational design and synthesis. 


\section{Results and discussion}

The geometries of the selected propellerenes (1-8) were optimized at PBE-D3(BJ)/6-31G(d,p) in their $C_{2}$ and $D_{3}$ conformation (Figure 2). Computed free Gibbs energies for the different conformers are in agreement with observed experimental preferences of the propellerenes, both in gas-phase (Figure 1) and solution phase (Table 1), with all ortho-substituted triphenylenes (1-4) preferring a $D_{3}$ conformation, ${ }^{2,3,27,42,43}$ and all benzenoid triphenylenes (5-8) preferring a $C_{2}$ conformation ${ }^{37,44-49}$ (trends were found consistent for both $\Delta \Delta G$ and $\Delta \Delta E$; see SI Table S4 for all data).

a)

Ortho-substituted propellerenes

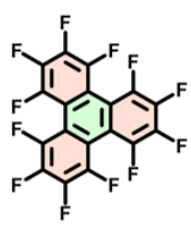

1

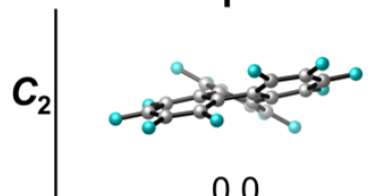

0.0

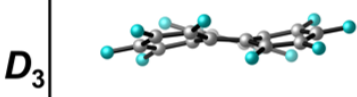

4.3

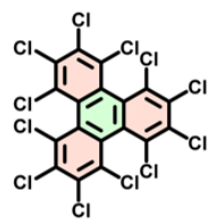

2

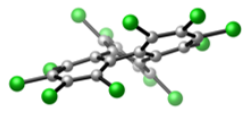

0.0

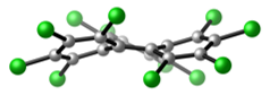

4.1

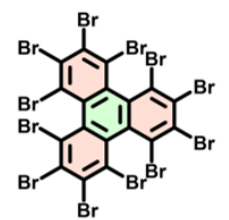

3

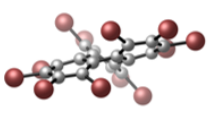

0.0

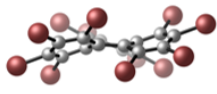

2.9
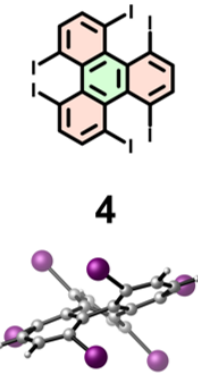

0.0

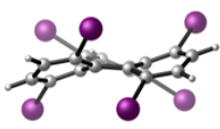

4.1

b)

Benzoid propellerenes

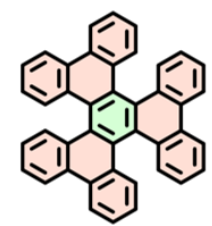

5

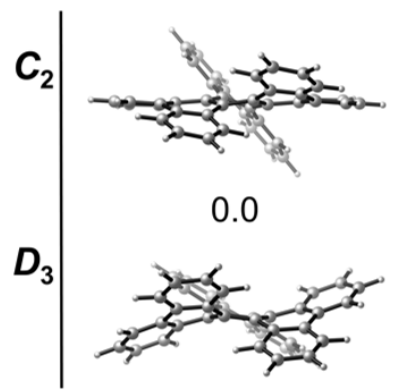

$-3.8$

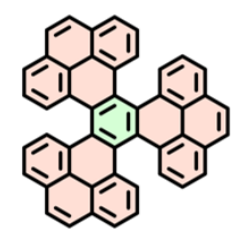

6

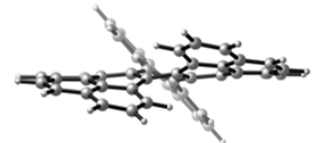

0.0

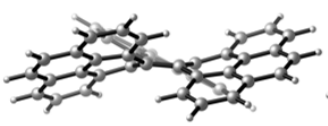

$-3.4$

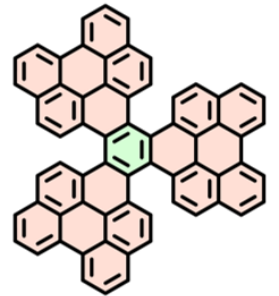

7

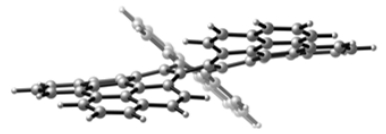

0.0

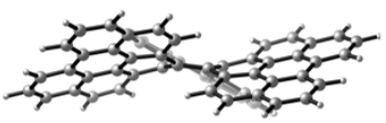

$-2.9$

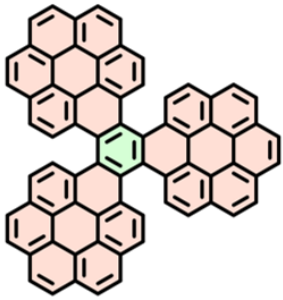

8

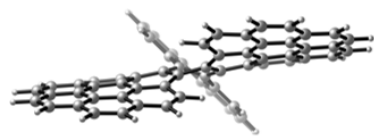

0.0

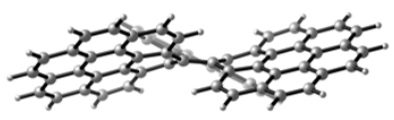

$-3.5$

Figure 2. Optimized geometries of a) ortho-substituted and b) benzenoid propellerenes the $C_{2}$ and $D_{3}$ conformation. Energy values below the structures are the Gibbs free energies $\left(\Delta G ; \mathrm{kcal} \mathrm{mol}^{-1}\right)$ computed at PBE$\mathrm{D} 3(\mathrm{BJ}) / 6-31 \mathrm{G}(\mathrm{d}, \mathrm{p})$ relative to the $C_{2}$ conformer. For computational details see SI. 
The ability of propellerenes to interconvert freely between their two conformations (i.e., the $C_{2}$ and $D_{3}$ conformation), was then derived from computed barrier heights for interconversion. With the exception of propellerene 1, the barrier height to interconversion, expressed with respect to the $C_{2}$ conformer (see Eq. 1), is sufficiently high $\left(\Delta G^{\ddagger}>23 \mathrm{kcal} \mathrm{mol}^{-1}\right)$ to prevent spontaneous isomerization at room temperature (Table 1). All computed interconversion barriers are in close agreement with the experimental values of identical or structurally similar compounds, validating our computational model.

Table 1. Computed and experimental conformational preference and barrier heights, relative to the $C_{2}$ conformer, for the interconversion to $D_{3}$ conformer $\left(\mathrm{kcal} \mathrm{mol}^{-1}\right)$ of benzenoid and ortho-substituted propellerenes. Computed data obtained at SMD(solvent)-PBE-D3(BJ)/6-31G(d,p). Solvent parameters were chosen as relevant for experimental conditions. Also see Table S1.

\begin{tabular}{lcrr}
\hline & $\begin{array}{c}\text { Preference } \\
\text { (exp.) }\end{array}$ & $\begin{array}{c}\Delta \Delta \boldsymbol{G} \\
\text { (comp.) }\end{array}$ & $\begin{array}{c}\Delta \mathbf{G} \neq \\
\text { (comp.) }\end{array}$ \\
\hline $\mathbf{1}$ & $C_{2}$ & 4.4 & $6.4^{c}$ \\
$\mathbf{2}$ & $C_{2}$ & 4.0 & $24.7^{b}$ \\
$\mathbf{3}$ & $C_{2}$ & 2.7 & $34.3^{a}$ \\
$\mathbf{4}$ & $C_{2}$ & 4.2 & $35.2^{d}$ \\
\hline $\mathbf{5}$ & $D_{3}$ & -3.6 & $25.1^{a}$ \\
$\mathbf{6}$ & $D_{3}$ & -3.1 & $25.8^{b}$ \\
$\mathbf{7}$ & $D_{3}$ & -2.9 & $23.3^{b}$ \\
$\mathbf{8}$ & $D_{3}$ & -3.4 & $28.7^{a}$ \\
\hline a $\mathrm{CHCl}_{3}, b \mathrm{PhMe}, c \mathrm{THF}, d \mathrm{DMSO}$, e DCM
\end{tabular}

We then wanted to understand the origin of the thermodynamic preference of the propellerenes and, in particular, the contrasting conformational preferences of the ortho-substituted and benzenoid families of propellerenes for a $C_{2}$ and $D_{3}$ conformation, respectively. We hypothesized the balance between the intrinsic preferences of the core and the wings of the propellerene to determine the most favorable conformation. To test this hypothesis, we employed the activation strain model (ASM; see SI for more full description of the computational method). ${ }^{53-59}$ This model considers the flexibility/rigidity of defined molecular fragments in a chemical system, as well as the ability of these fragments to interact with one another. Thus, the total energy, $\Delta E_{\text {tot, }}$ of a system is decomposed into a total strain of the molecular fragments and interaction energy term, $\Delta E_{\text {strain }}$ and $\Delta E_{\text {int }}$ as:

$$
\Delta E_{\text {tot }}=\Delta E_{\text {strain }}+\Delta E_{\text {int }}
$$

Here, the strain energy, $\Delta E_{\text {strain }}$, encompasses the penalty that needs to be paid in order to deform the molecular fragments from an initial state (here the $C_{2}$ conformation) to another state (here the $D_{3}$ conformation). The interaction energy term, $\Delta E_{\text {int, }}$ accounts for all the interactions that occur between these two molecular fragments in their different states (including both covalent and non-covalent interactions).

In the original ASM model, these different states of the molecular fragments are projected unto a reaction coordinate, typically describing bimolecular reactions, ${ }^{60-64}$ although studies concerning dyotropic and cyclization reactions of unimolecular systems are also known in the literature. ${ }^{65,66}$ However, in our case, we must extend the ASM to allow tracking of the changes in the strain experienced by different parts of the same molecule, as well as the interaction between these parts, in two distinct conformations ( $c f$. Scheme 1). Therefore, to apply the ASM method in an insightful manner, the core and wings as defined above were designated as separate fragments. These were then cleaved homolytically, to yield (uncharged) fragments with spins of +6 and -6 on the core and wings, 
respectively (Scheme 2). The analysis was then performed on the spin-restricted fragments in their spinunrestricted electronic configuration. This approach is similar to that used by Krenske et al. ${ }^{67-69}$ and others,$^{70}$ but differs in that in our model, radicals are not capped and fragment geometries are not relaxed.

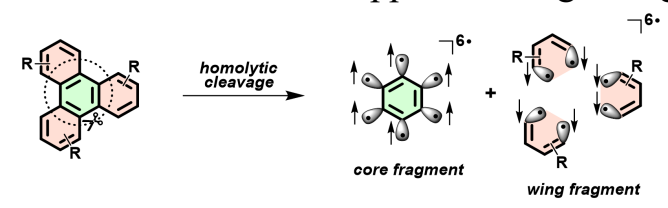

Scheme 2. Schematic representation of the homolytic cleavage of propellerenes to yield hexaradical core and wing fragments.

In our approach, the total energy of the propellerene system is expressed as the sum of the strain experienced by the core $\left(\Delta E_{\text {strain }}^{\text {core }}\right)$ and wing fragments $\left(\Delta E_{\text {strain }}^{\text {wing }}\right)$, and the interaction between the fragments $\left(\Delta E_{\text {int }}\right)$. Equation 1 can thus be re-written as:

$$
\Delta E_{\mathrm{tot}}=\left(\Delta E_{\text {strain }}^{\text {core }}+\Delta E_{\text {strain }}^{\text {wing }}\right)+\Delta E_{\text {int }}
$$

The interaction energy term in Eq. 2 was further decomposed using a canonical energy decomposition analysis (EDA) and analyzed in terms of the quantitative Kohn-Sham molecular orbital theory (KS-MO) which is described in detail in the Supplementary Information; for data see Table S4. ${ }^{71-73}$ In our analysis, all the energy terms are expressed with respect to the $C_{2}$ conformation (Eq. 3). As such, positive values of $\Delta \Delta E$ (as well as $\Delta \Delta G$ and $\Delta \Delta V$ ) indicate a preference for the $C_{2}$ conformation, whereas negative values represent a preference for the $D_{3}$ conformation.

$$
\Delta \Delta E=\Delta E_{\text {tot }}^{D_{3}}-\Delta E_{\text {tot }}^{C_{2}}
$$

Table 2 summarizes the results of the activation strain analysis (ASA). Three general trends can be observed. First, for all propellerenes under consideration here (1-8), the total strain energy is always more destabilizing in the more distorted $C_{2}$ conformation. Second, decomposition of the total strain energy terms into the strain energies of the individual fragments, i.e. $\Delta E_{\text {strain }}^{\text {wing }}$ and $\Delta E_{\text {strain }}^{\text {core }}$, reveals that the wing strain is more destabilizing in the distorted $C_{2}$ conformation, whereas the core is always less destabilizing in the $C_{2}$ conformation. In fact, we find that the preference of the wings for a $D_{3}$ conformation is always larger in magnitude than the preference of the core for a $C_{2}$ conformation. Lastly, like the core strain, the interaction energy, $\Delta E_{\text {int }}$, which describes the interaction between the core and the wings, is also found always more stabilizing in the $C_{2}$ conformation for all propellerenes. We will show, that the $\Delta E_{\text {int }}$ term plays a prominent role in the conformational preference for the different propellerene families (vide infra).

Next, we turn to explaining (i) the origin of the preferences of the different fragments and (ii) the influence of the substituents attached to the core (i.e. ortho-substituted versus benzenoid) on the conformational preference. Before doing so, it is important to reiterate that the wings in a propellerene molecule can only adopt two, geometrically possible, relative orientations; which gives rise to either a $C_{2}$ or $D_{3}$ symmetrical arrangement. The core, which is formed from the joining together of these wings, therefore adopts either a twist boat or chair-like geometry, respectively (see Scheme 1 and Figure 4). In other words, the arrangement of the wings dictates the shape of the core. 
Table 2. Activation strain and energy decomposition analysis of propellerenes 1-8. All energies $\left(\mathrm{kcal} \mathrm{mol}^{-1}\right)$ are computed at ZORA-PBE-D3(BJ)/TZ2P//PBE-D3(BJ)/6-31G(d,p) and are reported with respect to the $C_{2}$ conformation. For all data see SI Table S4.

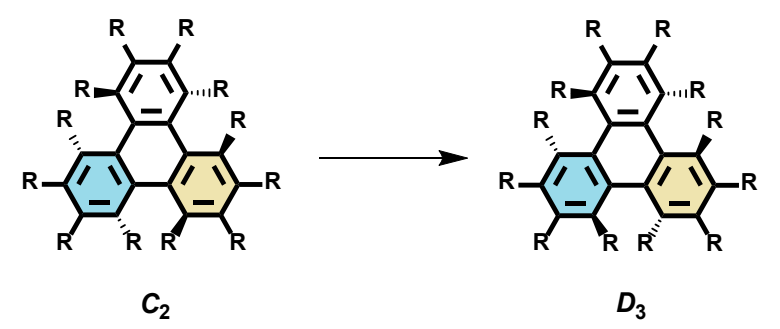

\begin{tabular}{rrrrrr}
\hline & $\Delta \Delta E$ & $\Delta \Delta E_{\text {strain }}^{\text {total }}$ & $\Delta \Delta E_{\text {strain }}^{\text {core }}$ & $\Delta \Delta E_{\text {strain }}^{\text {wing }}$ & $\Delta \Delta E_{\text {int }}$ \\
\hline $\mathbf{1}$ & 5.0 & 1.2 & 0.9 & 0.4 & 3.8 \\
$\mathbf{2}$ & 4.3 & -2.3 & 1.9 & -4.2 & 6.5 \\
$\mathbf{3}$ & 3.1 & -3.1 & 2.5 & -5.6 & 6.3 \\
$\mathbf{4}$ & 4.2 & -3.2 & 3.3 & -6.4 & 7.3 \\
\hline $\mathbf{5}$ & -3.6 & -6.2 & 3.0 & -8.2 & 3.0 \\
$\mathbf{6}$ & -3.1 & -5.0 & 1.3 & -6.3 & 1.9 \\
$\mathbf{7}$ & -2.9 & -4.8 & 2.1 & -6.9 & 1.9 \\
$\mathbf{8}$ & -3.4 & -5.1 & 1.0 & -6.1 & 1.7 \\
\hline
\end{tabular}

Wings fragment. Being aromatic, the wings of propellerenes have an intrinsic preference to be flat, which is quantitatively reflected in their general preference for a $D_{3}$ conformation (Table 2). Generally, as highlighted in Figure 3, in the $D_{3}$ conformation, the wings are by and large planar, and thus unstrained, whereas steric congestion of the wings in the $C_{2}$ conformation necessitates a significant distortion from planarity. Perfluorotriphenylene 1 constitutes the smallest triphenylene-class propellerene to still exhibit chiral behavior. The small size of the fluorine atoms, however, means its wings experience only minor steric hindrance, which is reflected in the absence of a strong preference for either a $C_{2}$ or $D_{3}$ conformation in any of the strain terms (for more information see Figure S3 and SI Table S6; c.f. Table 1). Upon increase of the size of the wing substituents, a notable preference for the less distorted $D_{3}$ conformation becomes apparent, with the ortho-substituted propellerenes (1-4) showing a trend as I > $\mathrm{Cl}>\mathrm{Br}>\mathrm{F}$, which can be directly traced back to the size of the halogens. For the benzenoid propellerenes no clear trends are observed, as introduction of additional benzene rings onto the ring does not significantly influence the steric clash experienced at the ortho-position. We additionally note that for propellerene 1, the wings are actually found to be more planar in the $C_{2}$ conformation than in the $D_{3}$ conformation, whereas it are the substituents attached thereto (i.e. the $\mathrm{F}$ atoms) that are bend more outof-plane (Figure S3 and SI Table S6). It is the balance between these two purely structural factors that results in the preference of the wings of $\mathbf{1}$ for the $C_{2}$ conformation. We note that this balancing game is only possible for ortho-substituted wings, due to the relative flexibility of the unitary benzene ring, whereas benzenoid extended wings are too rigid, and will therefore always show a $D_{3}$ preference (vide infra). 


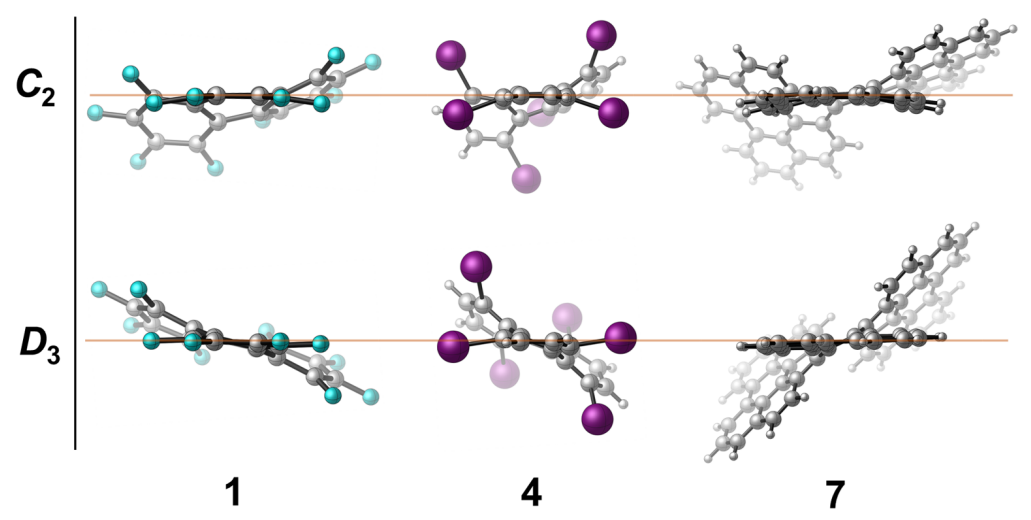

Figure 3. Side-view of propellerene 1, 4 and 7. Brown lines highlight the different extents of deformation (i.e. out-of-plane bending of the wings) in the $C_{2}$ conformation, compared to their relative planarity in the $D_{3}$ conformation.

Radial bonds. Regardless of conformation, the radial bonds which connect the propellerene wings to the core are significantly stretched compared to typical bond lengths found in planar PAHs. This is a direct result of the steric clash experienced by the propellerene wings. For all propellerenes, the radial bonds prefer to reside in the more distorted $C_{2}$ conformation, in which they are slightly shorter than in the $D_{3}$ conformation (SI Table S3). Having shorter bonds, however, means the wings approach each other at closer distances, necessitating them to distort to minimize steric clash (vide infra). In addition, careful inspection of the propellerenes geometries revealed that in the $C_{2}$ conformation, the twist boatlike geometry of the core allows two of the wings to join in an almost coplanar fashion, whereas in the $D_{3}$ conformation the chair-like geometry of the ring dictates a more skewed attachment of the wings (Figure 4; highlighted pink and yellow). To verify whether the more stabilizing interaction energy between the wings and core ( $\left.\Delta E_{\text {int }}\right)$ for the more distortive $C_{2}$ (Table 2) originates solely from differences in bond length, or whether the geometry of the bond attachment is also of importance, a numerical experiment was performed. The radial bonds of propellerenes in their $D_{3}$ conformer were artificially shortened to be of the same length as in their corresponding $C_{2}$ conformer (see SI Table 5 for all data). Identical trends in terms of interaction energy were observed, however the absolute differences became smaller, indicating that the more stabilizing interaction energy found for the $C_{2}$ (Table 2) originates from both the radial bond lengths and the way in which the radial bonds are attached, in which the former is more important. Importantly, by artificially shortening the radial bonds in the $D_{3}$ conformation, the wing strain becomes more destabilizing, which can be directly traced back to the increase in steric hindrance between the wings (see Table S5). This thus reinforces the notion that in the $D_{3}$ conformation it is not possible for the wings to approach each other at a closer distance, and hence necessitates longer radial bonds, and concomitantly a less stabilizing interaction energy.

a)

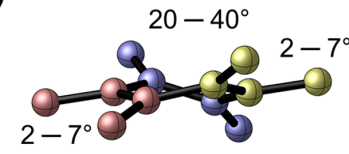

$C_{2}$ b)

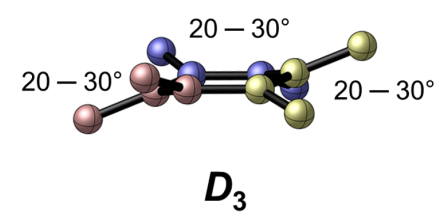

Figure 4. Representative geometries of the core fragment and radial bonds in the $C_{2}$ and $D_{3}$ conformation with key dihedral angles color-coded (pink, blue, yellow), with the range of angles found in 1-8. This illustrates the different ways in which the wings are adjoined to the core in the different conformations. For all dihedral angles see SI Table S2. 
Core fragment. Like the radial bonds, the bond lengths found in the cores of propellerenes are always longer compared to typical bond lengths found in planar PAHs. Again, this is a direct result of the steric interactions experienced between the wings of the propellerenes, i.e. longer bonds within the core effectively place the wings further apart from each other. Conversely with the radial bonds, the $\mathrm{C}-\mathrm{C}$ bonds within the core of propellerenes are always longer in the $C_{2}$ conformation than in the $D_{3}$ conformation; i.e. the core of the $C_{2}$ conformer is larger than that of the $D_{3}$ conformer (SI Table S3). As an exemplary experiment, when the bare core was allowed to relax, without constrains, bond lengths became shorter and a planar, benzene-like molecule was obtained. Thus, the core of a propellerene molecule ideally wants to have shorter $\mathrm{C}-\mathrm{C}$ bonds, but, in the context of a propellerene is geometrically prohibited from doing so by the presence of the wings. Despite the longer bond lengths, the core strain is less destabilizing in this conformation. To verify whether this preference is due to the bond lengths or whether there is an intrinsic preference for the skew-boat conformation, we performed a numerical experiment. Bond lengths of propellerene core fragments in their $C_{2}$ and $D_{3}$ conformation were constrained and all dihedral angles were artificially set to $0^{\circ}$, forcing the rings to become flat. Surprisingly, absolute differences in total energy diminished, and even slightly shifted in favor of the $D_{3}$ conformation (SI Table S7). These results support the notion that the core intrinsically wants to adopt the skew-boat like structure over the flattened chair (Figure 4). These findings further reinforce the notion that the conformation of the core is enforced by the wings, rather than the other way around..$^{20,35,74-}$ 79
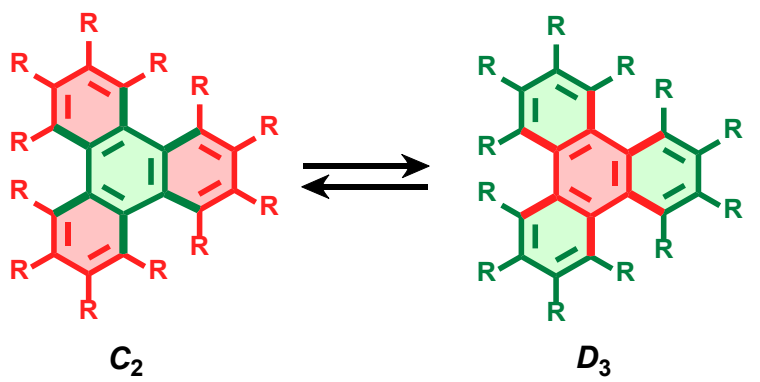

Figure 5. Schematic overview of the $C_{2} \rightleftarrows D_{3}$ interconversion of propellerenes to graphically illustrate that the wings of propellerenes prefer to adopt the $D_{3}$ conformation, whereas the radial bonds and core want to reside in a more distorted $C_{2}$ conformation. The green color indicates that the fragment prefers the specific conformation, while the opposite is true for the red color. Radial bonds drawn in bold.

Combined, based on our ASA, we find that the integral propellerene parts all share the same characteristics in both propellerene families. Importantly, it is the magnitude of the interaction energy between the core and the wings that varies most between the two families, and ultimately lays at the heart of the sharp contrast in conformational preference between ortho- and benzenoid-substituted propellerenes. The magnitude of the interaction energy is, in turn, dictated by the flexibility of the propellerene wings. When wings are relatively flexible (i.e. ortho-substituted propellerenes) they are able to adopt a more distorted $C_{2}$ conformation, allowing for shorter radial bonds between the core and the wings, with concomitantly more a stabilizing interaction energy. In contrast, more rigid wings cannot deform sufficiently (i.e. benzenoid-substituted propellerenes), and thus necessitate longer radial bonds to avoid steric clash between the wings, and incurring less stabilizing interaction energy (Figure 5). This interplay between the flexibility of the wings and length of the radial bonds is graphically illustrated in Figure 6. There it is apparent that for ortho-substituted propellerenes, conversion from the $C_{2}$ conformation to the $D_{3}$ conformation is associated with flatter wings (favorable) but also longer radial bonds (unfavorable), whereas for the benzenoid-substituted propellerenes there is hardly any change in radial bond length during interconversion, and the preference for a $D_{3}$ conformation is thus purely dictated by the relative planarity of the wings in the two conformations. 


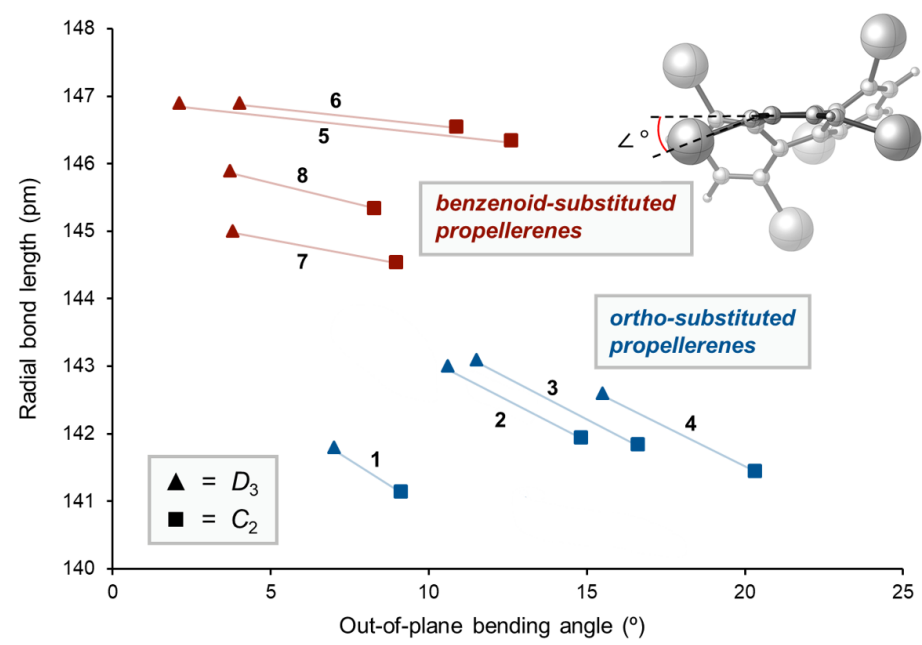

Figure 6. Plot of the average radial bond length as a function of the average bending angle of wings of propellerenes 1-8 in the $C_{2}$ and $D_{3}$ conformation. Compounds and conformations are groups as: benzenoid- $C_{2}$ (brown squares), benzenoid- $D_{3}$ (brown triangles), ortho- $C_{2}$ (blue squares) and ortho- $D_{3}$ (blue triangles).. The definition of the out-of-plane bending angle is illustrated in the top right corner.

Model validation. To validate our hypothesis that the flexibility of the wing is the dominant factor in driving the conformational preference of propellerenes, additional molecules (9-11) were selected and analysed using the present model (Table 3). These molecules all have alkyl substituents on the ortho position. It is observed that compound $\mathbf{1 0}$ follows the general trend observed for the halogenated propellerenes (Table 2), i.e. the wings prefer a $D_{3}$ conformation, whereas the core and radial bonds prefer a $C_{2}$ conformation, the latter term of which is able to overcompensate the wing strain term. Compounds 9 and 11, however, behave more like perfluorotriphenylene 1 in that all terms, including the wings, prefer a $C_{2}$ conformation. This is accounted for by the present model, as the flexibility of the substituents in 9 and $\mathbf{1 1}$ results in negligible conformational preference of the wings, as a result of which the preferences of the radial bonds and core become the dominant factor, and a $C_{2}$ conformation is adopted. Compound $\mathbf{1 1}$ is a particularly interesting case, being the hydrogenated variant of $\mathbf{2}$, which prefers a $D_{3}$ conformation. In other words, upon hydrogenation of $\mathbf{2}$, the ortho-carbons of the wings change from $s p^{2}$ to $s p^{3}$, making the wings substantially more flexible, causing a shift in conformational preference from $D_{3}$ to $C_{2}$.

Table 3. Activation strain and energy decomposition analysis of propellerenes 9-11. All energies $\left(\mathrm{kcal} \mathrm{mol}^{-1}\right)$ are computed at ZORA-PBE-D3(BJ)/TZ2P//PBE-D3(BJ)/6-31G(d,p) and are reported with respect to the $C_{2}$ conformation.

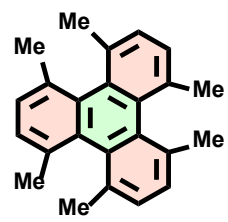

9

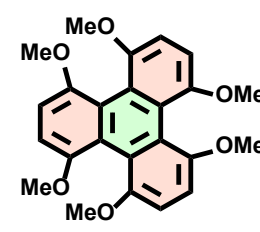

10

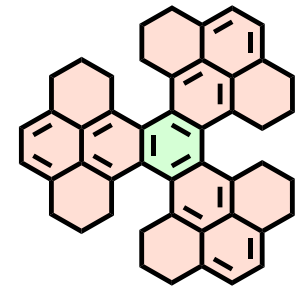

11

\begin{tabular}{rrrrrc}
\hline & $\Delta \Delta E$ & $\Delta \Delta E_{\text {strain }}^{\text {total }} \Delta \Delta E_{\text {strain }}^{\text {core }} \Delta \Delta E_{\text {strain }}^{\text {wing }}$ & $\Delta \Delta E_{\text {int }}$ \\
\hline $\mathbf{9}$ & 4.9 & 3.3 & 3.3 & 0.0 & 1.6 \\
10 & 3.3 & -4.3 & 2.7 & -7.0 & 7.6 \\
11 & 4.2 & 2.8 & 2.4 & 0.4 & 1.4 \\
\hline
\end{tabular}




\section{Conclusions}

The conformational preference of propeller-shaped polycyclic aromatic hydrocarbons has been the topic of heavy debate. Triphenylene-class propellerenes with substituents on the ortho-position prefer to adopt a conformation with a $C_{2}$ conformation, whereas those with benzenoid substituents prefer to adopt a $D_{3}$ conformation. Herein, we provide a comprehensible framework to understand and rationalize the driving forces behind the conformational preference of a range of structurally diverse propellerenes.

The origin of the thermodynamic preference of propellerenes could be quantified using a novel adaptation of the activation strain model (ASM). By strategically fragmenting propellerene molecules, we find that both the core and wings of propellerenes desire to be flat, however, their merger necessitates significant deformation, away from their ideal geometry, to minimize steric interactions between the wings. We found the desire of the wings for the $D_{3}$ conformation to always be greater than the desire of the core for a $C_{2}$ conformation. Attention was therefore shifted to the radial bonds, which connect the core and wings together. These were also found to always prefer a $C_{2}$ conformation and, in the case of ortho-substituted propellerenes, are even able to overrule the preference of the wings. We were ultimately able to rationalize that the balance at play in propellerenes is between the extent to which the wings can bend and the radial bonds can contract. When wings are flexible, a $C_{2}$ conformation will be preferred, when wings are rigid, a $D_{3}$ conformation will always be preferred.

To conclude, these findings will equip experimentalists with the insight to understand and rationalize the trends in conformational behavior of propellerenes and allow the tailor-made design of novel sterically congested structures. Although applied here only to propellerenes, the present methodology is broadly applicable and will open up new avenues not only in the field of PAH research but in the field of physical chemistry at large, and should prove useful in the rational design of novel functional constructs.

\section{Acknowledgments}

This work is supported by an NWO VIDI (Vidi 723.013.007) grant and ERC Proof of Concept NANOPORE (no. 780004) grant awarded to G. F. S. This work was carried out on the Dutch national e-infrastructure with the support of SURF Cooperative via an NWO-Rekentijd grant 17569 (to T.H. and J.D.C.C.). We kindly acknowledge Mark Somers of Leiden University for technical support.

\section{ORCID}

Alex van der Ham: 0000-0001-5018-3309

Thomas Hansen: 0000-0002-6291-1569

Dmitri V. Filippov: 0000-0002-6978-7425

Herman S. Overkleeft: 0000-0001-6976-7005

Trevor A. Hamlin: 0000-0002-5128-1004

Grégory F. Schneider: 0000-0002-3156-4525 


\section{Table of Contents}

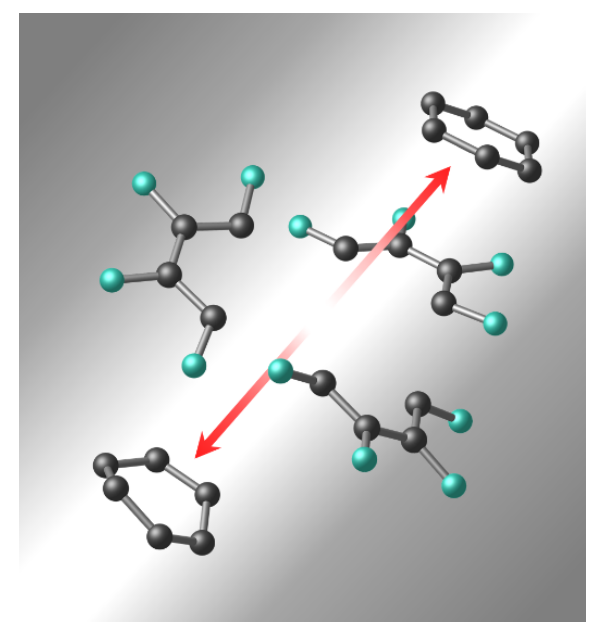

Mix and match! A novel approach to understand the origin of the conformation preference of propellershaped molecules is presented. By computationally disecting propellerenes, it is found that their conformational preference ultimately originates from the flexibility of their wings. 


\section{References}

(1) Tanaka, H.; Kato, Y.; Fujiki, M.; Inoue, Y.; Mori, T. Combined Experimental and Theoretical Study on Circular Dichroism and Circularly Polarized Luminescence of Configurationally Robust $D_{3}$-Symmetric Triple Pentahelicene. J. Phys. Chem. A 2018, 122 (37), 7378.

(2) Berezhnaia, V.; Roy, M.; Vanthuyne, N.; Villa, M.; Naubron, J.; Rodriguez, J.; Coquerel, Y.; Gingras, M. Chiral Nanographene Propeller Embedding Six Enantiomerically Stable [5]Helicene Units. J. Am. Chem. Soc. 2017, 139 (51), 18508.

(3) Hosokawa, T.; Takahashi, Y.; Matsushima, T.; Watanabe, S.; Kikkawa, S.; Azumaya, I.; Tsurusaki, A.; Kamikawa, K. Synthesis, Structures, and Properties of Hexapole Helicenes: Assembling Six [5]Helicene Substructures into Highly Twisted Aromatic Systems. J. Am. Chem. Soc. 2017, 139 (51), 18512.

(4) Rickhaus, M.; Mayor, M.; Juríček, M. Chirality in Curved Polyaromatic Systems. Chem. Soc. Rev. 2017, 46 (6), 1643.

(5) Rickhaus, M.; Mayor, M.; Juríček, M. Strain-induced Helical Chirality in Polyaromatic Systems. Chem. Soc. Rev. 2016, 45 (6), 1542.

(6) Kato, K.; Osuka, A. Propeller-shaped Semi-fused Porphyrin Trimers: Molecular-symmetrydependent Chiroptical Response. Chem. Eur. J. 2020, 26 (45), 10217.

(7) Hasan, M.; Borovkov, V. Helicene-based Chiral Auxiliaries and Chirogenesis. Symmetry 2018, $10(1), 10$.

(8) Hembury, G. A.; Borovkov, V. V.; Inoue, Y. Chirality-sensing Supramolecular Systems. Chem. Rev. 2008, 108 (1), 1.

(9) Yang, Y.; Da Costa, R. C.; Fuchter, M. J.; Campbell, A. J. Circularly Polarized Light Detection by a Chiral Organic Semiconductor Transistor. Nat. Photonics 2013, 7 (8), 634.

(10) Chen, F.; Tanaka, T.; Hong, Y. S.; Mori, T.; Kim, D.; Osuka, A. Closed Pentaaza [9]helicene and Hexathia [9]/[5]helicene: Oxidative Fusion Reactions of ortho-Phenylene-Bridged Cyclic Hexapyrroles and Hexathiophenes. Angew. Chem. 2017, 129 (46), 14880.

(11) Hashimoto, S.; Nakatsuka, S.; Nakamura, M.; Hatakeyama, T. Construction of a Highly Distorted Benzene Ring in a Double Helicene. Angew. Chem. Int. Ed. 2014, 53 (51), 14074.

(12) Sakamaki, D.; Kumano, D.; Yashima, E.; Seki, S. A Facile and Versatile Approach to Double N-Heterohelicenes: Tandem Oxidative C-N Couplings of N-Heteroacenes via Cruciform Dimers. Angew. Chem. 2015, 127 (18), 5494.

(13) Ferreira, M.; Naulet, G.; Gallardo, H.; Dechambenoit, P.; Bock, H.; Durola, F. A Naphtho-Fused Double [7]Helicene from a Maleate-Bridged Chrysene Trimer. Angew. Chem. Int. Ed. 2017, 56 (12), 3379.

(14) Tanaka, H.; Ikenosako, M.; Kato, Y.; Fujiki, M.; Inoue, Y.; Mori, T. Symmetry-based Rational Design for Boosting Chiroptical Responses. Commun. Chem. 2018, 1 (1), 1.

(15) Liu, X.; Yu, P.; Xu, L.; Yang, J.; Shi, J.; Wang, Z.; Cheng, Y.; Wang, H. Synthesis for the Mesomer and Racemate of Thiophene-based Double Helicene under Irradiation. J. Org. Chem. 2013, 78 (12), 6316.

(16) Wang, X. Y.; Wang, X. C.; Narita, A.; Wagner, M.; Cao, X. Y.; Feng, X.; Müllen, K. Synthesis, Structure, and Chiroptical Properties of a Double [7]heterohelicene. J. Am. Chem. Soc. 2016, 138 (39), 12783.

(17) Katayama, T.; Nakatsuka, S.; Hirai, H.; Yasuda, N.; Kumar, J.; Kawai, T.; Hatakeyama, T. Twostep Synthesis of Boron-fused Double Helicenes. J. Am. Chem. Soc. 2016, 138 (16), 5210.

(18) Hu, Y.; Wang, X. Y.; Peng, P. X.; Wang, X. C.; Cao, X. Y.; Feng, X.; Müllen, K.; Narita, A. Benzo-Fused Double [7]Carbohelicene: Synthesis, Structures, and Physicochemical Properties. Angew. Chem. Int. Ed. 2017, 56 (12), 3374.

(19) Lütke Eversloh, C.; Liu, Z.; Müller, B.; Stangl, M.; Li, C.; Müllen, K. Core-Extended Terrylene Tetracarboxdiimide: Synthesis and Chiroptical Characterization. Org. Lett. 2011, 13 (20), 5528.

(20) Luo, J.; Xu, X.; Mao, R.; Miao, Q. Curved Polycyclic Aromatic Molecules that are $\pi$ isoelectronic to Hexabenzocoronene. J. Am. Chem. Soc. 2012, 134 (33), 13796. 
(21) Peña, D.; Cobas, A.; Perez, D.; Guitián, E.; Castedo, L. Dibenzo[a,o]phenanthro[3, 4-s]pycene, a Configurationally Stable Double Helicene: Synthesis and Determination of Its Conformation by NMR and GIAO Calculations. Org. Lett. 2003, 5 (11), 1863.

(22) Kashihara, H.; Asada, T.; Kamikawa, K. Synthesis of a Double Helicene by a PalladiumCatalyzed Cross-Coupling Reaction: Structure and Physical Properties. Chem. Eur. J. 2015, 21 (17), 6523.

(23) Fujikawa, T.; Mitoma, N.; Wakamiya, A.; Saeki, A.; Segawa, Y.; Itami, K. Synthesis, Properties, and Crystal Structures of $\pi$-extended Double [6]helicenes: Contorted Multidimensional Stacking Lattice. Org. Biomol. Chem. 2017, 15 (21), 4697.

(24) Fujikawa, T.; Segawa, Y.; Itami, K. Synthesis, Structures, and Properties of $\pi$-extended Double Helicene: A Combination of Planar and Nonplanar $\pi$-systems. J. Am. Chem. Soc. 2015, 137 (24), 7763.

(25) Saito, H.; Uchida, A.; Watanabe, S. Synthesis of a Three-Bladed Propeller-Shaped Triple [5] Helicene. J. Org. Chem. 2017, 82 (11), 5663.

(26) Meng, D.; Fu, H.; Xiao, C.; Meng, X.; Winands, T.; Ma, W.; Wei, W.; Fan, B.; Huo, L.; Doltsinis, N. L. Three-bladed Rylene Propellers with Three-dimensional Network Assembly for Organic Electronics. J. Am. Chem. Soc. 2016, 138 (32), 10184.

(27) Pradhan, A.; Dechambenoit, P.; Bock, H.; Durola, F. Twisted Polycyclic Arenes by Intramolecular Scholl reactions of $C_{3}$-symmetric Precursors. J. Org. Chem. 2013, 78 (6), 2266.

(28) Bennett, M. A.; Kopp, M. R.; Wenger, E.; Willis, A. C. Generation of Nickel(0) Aryne and Nickel(II) Biphenyldiyl Complexes via in situ Dehydrohalogenation of Arenes. Molecular structures of $\left[\mathrm{Ni}\left(2,2^{\prime}-\mathrm{C}_{6} \mathrm{H}_{4} \mathrm{C}_{6} \mathrm{H}_{4}\right)(\mathrm{dcpe})\right]$ and $C_{2}$-hexabenzotriphenylene. J. Organomet. Chem 2003, 667 (1-2), 8 .

(29) Zetong, M.; Thorsten, W.; Ningning, L.; Dong, M.; Wei, J.; Zhaohui, W. A $C_{2}$-symmetric Triple [5]helicene Based on N-annulated Triperylene Hexaimide for Chiroptical Electronics. Sci. China Chem. 2019, 63 (2), 208.

(30) Hagen, S.; Bratcher, M. S.; Erickson, M. S.; Zimmermann, G.; Scott, L. T. Novel Syntheses of Three $\mathrm{C}_{30} \mathrm{H}_{12}$ Bowl-Shaped Polycyclic Aromatic Hydrocarbons. Angew. Chem. Int. Ed. 1997, $36(4), 406$.

(31) Hagen, S.; Scott, L. T. A Convenient Synthesis of Benzo[c]naphtho[2,1-p]chrysene. J. Org. Chem. 1996, 61 (20), 7198.

(32) Fujikawa, T.; Segawa, Y.; Itami, K. Synthesis and Structural Features of Quadruple Helicenes: Highly Distorted $\pi$ Systems Enabled by Accumulation of Helical Repulsions. J. Am. Chem. Soc. 2016, 138 (10), 3587.

(33) Mori, T. Chiroptical Properties of Symmetric Double, Triple, and Multiple Helicenes. Chem. Rev. 2021, 121 (4), 2373.

(34) Shibata, K.; Kulkarni, A. A.; Ho, D. M.; Pascal Jr., R. A. The Pursuit of Perchlorotriphenylene. J. Org. Chem. 1995, 60 (2), 428.

(35) Barnett, L.; Ho, D. M.; Baldridge, K. K.; Pascal, R. A. The Structure of Hexabenzotriphenylene and the Problem of Overcrowded " $D_{3 \mathrm{~h}}$ " Polycyclic Aromatic Compounds. J. Am. Chem. Soc. 1999, 121 (4), 727.

(36) Balaban, A. T.; Randić, M. Structural approach to aromaticity and local aromaticity in conjugated polycyclic systems; Springer, 2011.

(37) Smith, V. B.; Massey, A. G. Perfluorophenyl Derivatives of the Elements-XXII: Perfluorotriphenylene. Tetrahedron 1969, 25 (22), 5495.

(38) Coe, P. L.; Stephens, R.; Tatlow, J. C. 622. Aromatic Polyfluoro-compounds. Part XI. Pentafluoro-phenyl-lithium and Derived Compounds. J. Chem. Soc. (Resumed) 1962, 3227.

(39) Falk, R. A. Hexafluorobenzene and Related Fluoroaromatic Compounds. Sperry Eng. Rev. 1963, 16, 24.

(40) Shibata, K.; Kulkarni, A. A.; Ho, D. M.; Pascal Jr., R. A. Perchlorotriphenylene. J. Am. Chem. Soc. 1994, 116 (13), 5983.

(41) In their study, Pascal et al. considered only the carbon framework when commenting on the relative planarity of the wings. In the present study, the out-of-plane bending of the ortho substituents is also included. 
(42) Zuzak, R.; Castro-Esteban, J.; Brandimarte, P.; Engelund, M.; Cobas, A.; Piątkowski, P.; Kolmer, M.; Pérez, D.; Guitián, E.; Szymonski, M. Building a 22-ring Nanographene by Combining In-solution and On-surface Syntheses. Chem. Commun. 2018, 54 (73), 10256.

(43) Peña, D.; Cobas, A.; Pérez, D.; Guitián, E.; Castedo, L. Kinetic Control in the PalladiumCatalyzed Synthesis of C2-Symmetric Hexabenzotriphenylene. A Conformational Study. Org. Lett. 2000, 2 (11), 1629.

(44) Naritomi, M.; Murofushi, H.; Nakashima, N. Dopants for a Perfluorinated Graded Index Polymer Optical Fiber. Bull. Chem. Soc. Jpn. 2004, 77 (11), 2121.

(45) Tan, Q.; Zhou, D.; Zhang, T.; Liu, B.; Xu, B. Iodine-doped Sumanene and Its Application for the Synthesis of Chalcogenasumanenes and Silasumanenes. Chem. Commun. 2017, 53 (74), 10279 .

(46) Wang, Y.; Stretton, A. D.; McConnell, M. C.; Wood, P. A.; Parsons, S.; Henry, J. B.; Mount, A. R.; Galow, T. H. 1,4,5,8,9,12-Hexamethyltriphenylene. A Molecule with a Flipping Twist. J. Am. Chem. Soc. 2007, 129 (43), 13193.

(47) Frampton, C. S.; MacNicol, D. D.; Rowan, S. J. Synthesis and Structural Properties of the first Dodecakis(aryloxy)triphenylenes. J. Mol. Struct. 1997, 405 (2-3), 169.

(48) Nishinaga, T.; Inoue, R.; Matsuura, A.; Komatsu, K. Formation of a Novel Arenium Ion from the Radical Cation of a Twisted Triphenylene Fully Annelated with Bicyclo[2.2.2]octene Units. Org. Lett. 2002, 4 (9), 1435.

(49) Li, Z.; Zhi, L.; Lucas, N. T.; Wang, Z. Triangle-shaped Polycyclic Aromatics Based on Tribenzocoronene: Facile Synthesis and Physical Properties. Tetrahedron 2009, 65 (17), 3417.

(50) Cosmo, R.; Sternhell, S. Steric effects. Inversion of 4,5-disubstituted 9,10Dihydrophenanthrenes. Aust. J. Chem. 1987, 40 (1), 35.

(51) Cosmo, R.; Hambley, T. W.; Sternhell, S. Skeletal Deformation in 4,5-disubstituted 9,10Dihydrophenanthrenes and 4,5-disubstituted Phenanthrenes. J. Org. Chem. 1987, 52 (14), 3119.

(52) Based on the absence of observable interconversion after 20 hrs at $70{ }^{\circ} \mathrm{C}$.

(53) Bickelhaupt, F. M.; Houk, K. N. Das Distortion/Interaction-Activation-Strain-Modell zur Analyse von Reaktionsgeschwindigkeiten. Angew. Chem. 2017, 129 (34), 10204.

(54) Bickelhaupt, F. M.; Houk, K. N. Analyzing Reaction Rates with the Distortion/interactionActivation Strain Model. Angew. Chem. Int. Ed. 2017, 56 (34), 10070.

(55) Fernández, I.; Bickelhaupt, F. M. The Activation Strain Model and Molecular Orbital Theory: Understanding and Designing Chemical Reactions. Chem. Soc. Rev. 2014, 43 (14), 4953.

(56) Bickelhaupt, F. M. Understanding Reactivity with Kohn-Sham Molecular Orbital Theory: E2$\mathrm{S}_{\mathrm{N}} 2$ Mechanistic Spectrum and Other Concepts. J. Comp. Chem. 1999, 20 (1), 114.

(57) Vermeeren, P.; van der Lubbe, S. C. C.; Guerra, C. F.; Bickelhaupt, F. M.; Hamlin, T. A. Understanding Chemical Reactivity Using the Activation Strain Model. Nature protocols 2020, $15(2), 649$.

(58) van Zeist, W.-J.; Bickelhaupt, F. M. The Activation Strain Model of Chemical Reactivity. Org. Biomol. Chem. 2010, 8 (14), 3118.

(59) Wolters, L. P.; Bickelhaupt, F. M. The Activation Strain Model and Molecular Orbital Theory. WIRES: Computational Molecular Science 2015, 5 (4), 324.

(60) Vermeeren, P.; Hansen, T.; Grasser, M.; Silva, D. R.; Hamlin, T. A.; Bickelhaupt, F. M. S 2 versus E2 Competition of $\mathrm{F}^{-}$and $\mathrm{PH}_{2}{ }^{-}$Revisited. J. Org. Chem. 2020, 85 (21), 14087.

(61) Vermeeren, P.; Hansen, T.; Jansen, P.; Swart, M.; Hamlin, T. A.; Bickelhaupt, F. M. A Unified Framework for Understanding Nucleophilicity and Protophilicity in the $\mathrm{S}_{\mathrm{N}} 2 / \mathrm{E} 2$ Competition. Chem.-Eur. J. 2020, 26 (67), 15538.

(62) Hansen, T.; Vermeeren, P.; Yoshisada, R.; Filippov, D. V.; Van Der Marel, G. A.; Codée, J. D. C.; Hamlin, T. A. How Lewis Acids Catalyze Ring-openings of Cyclohexene Oxide. J. Org. Chem. 2021, 86 (4), 3565.

(63) Hamlin, T. A.; Hansen, T.; Vermeeren, P.; Bickelhaupt, F. M. Origin of the $\alpha$-Effect in $\mathrm{S}_{\mathrm{N}} 2$ Reactions. Angew. Chem. Int. Ed. 2021.

(64) Vermeeren, P.; Hamlin, T. A.; Bickelhaupt, F. M. Chemical Reactivity From an Activation Strain Perspective. Chem. Commun. 2021.

(65) Fernández, I.; Bickelhaupt, F. M.; Cossío, F. P. Ene-ene-yne Reactions: Activation Strain Analysis and the Role of Aromaticity. Chem. Eur. J. 2014, 20 (34), 10791. 
(66) Fernández, I.; Bickelhaupt, F. M.; Cossío, F. P. Type-I Dyotropic Reactions: Understanding Trends in Barriers. Chem. Eur. J. 2012, 18 (39), 12395.

(67) Krenske, E. H.; Houk, K. N.; Holmes, A. B.; Thompson, J. Entropy versus Tether Strain Effects on Rates of Intramolecular 1,3-dipolar Cycloadditions of N-alkenylnitrones. Tet. Lett. 2011, 52 (17), 2181.

(68) Krenske, E. H.; Davison, E. C.; Forbes, I. T.; Warner, J. A.; Smith, A. L.; Holmes, A. B.; Houk, K. N. Reverse Cope Elimination of Hydroxylamines and Alkenes or Alkynes: Theoretical Investigation of Tether Length and Substituent Effects. J. Am. Chem. Soc. 2012, 134 (4), 2434.

(69) Krenske, E. H.; Perry, E. W.; Jerome, S. V.; Maimone, T. J.; Baran, P. S.; Houk, K. N. Why a Proximity-Induced Diels-Alder Reaction is So Fast. Org. Lett. 2012, 14 (12), 3016.

(70) Gold, B.; Shevchenko, N. E.; Bonus, N.; Dudley, G. B.; Alabugin, I. V. Selective Transition State Stabilization via hyperconjugative and conjugative assistance: stereoelectronic concept for copper-free click chemistry. J. Org. Chem. 2011, 77 (1), 75.

(71) Bickelhaupt, F. M.; Baerends, E. J.; John Wiley and Sons New York, NY, USA, 2000; Vol. 15.

(72) Van Meer, R.; Gritsenko, O. V.; Baerends, E. J. Physical Meaning of Virtual Kohn-Sham Orbitals and Orbital Energies: an Ideal Basis for the Description of Molecular Excitations. $J$. Chem. Theory Comput. 2014, 10 (10), 4432.

(73) a) Zhao, L.; von Hopffgarten, M.; Andrada, D. M. WIREs Comput. Mol. Sci. 2018, 8, e1345; The Chemical Bond. Fundamental Aspects of Chemical Bonding; b) G. Frenking, FM Bickelhaupt. The EDA Perspective of Chemical Bonding in The Chemical Bond. Fundamental Aspects of Chemical Bonding 2014, 4, 121.

(74) Although the exact reason for the preference of the core for a $C_{2}$ conformation could not be elucidated, these findings corroborate those made by others which found the central ring of propellerenes to be poorly or even non-aromatic; i.e. aromaticity and thus a drive to planarity are not significant factors in determining the conformational preference of the core in the context of a propellerene molecule.

(75) Fernandez, I.; Frenking, G. Direct Estimate of Conjugation and Aromaticity in Cyclic Compounds with the EDA Method. Faraday Discuss. 2007, 135, 403.

(76) Pierrefixe, S. C. A. H.; Bickelhaupt, F. M. Aromaticity: Molecular-Orbital Picture of an Intuitive Concept. Chem. Eur. J. 2007, 13 (22), 6321.

(77) Roy, M.; Berezhnaia, V.; Villa, M.; Vanthuyne, N.; Giorgi, M.; Naubron, J. V.; Poyer, S.; Monnier, V.; Charles, L.; Carissan, Y. Stereoselective Syntheses, Structures, and Properties of Extremely Distorted Chiral Nanographenes Embedding Hextuple Helicenes. Angew. Chem. Int. Ed. 2020, 59 (8), 3264.

(78) Antić, M.; Furtula, B.; Radenkovic, S. Aromaticity of Nonplanar Fully Benzenoid Hydrocarbons. J. Phys. Chem. A 2017, 121 (18), 3616.

(79) Hosokawa, T.; Tsurusaki, A.; Kamikawa, K. Assembly of [5]Helicene Subunits by PalladiumCatalyzed Reactions: Synthesis, Structures, Properties, and Theoretical Study of Multiple Helicenes. J. Synth. Org. Chem. Jpn. 2020, 78 (11), 1013. 